\title{
Forum \\ 8. Three Distinctions about Concepts and Categorization
}

\author{
EDWARD E. SMITH
}

Some of the problems raised in the target text for this Forum may be alleviated by a consideration of three distinctions about concepts and categories. These include the distinctions between: (1) metaphysical versus epistemological accounts of concepts, (2) the core versus the identification procedure of a concept, and (3) different kinds of concepts. I consider these distinctions in turn.

\section{Metaphysical versus Epistemological Accounts of Concepts}

The difference between metaphysical and epistemological approaches to concepts is crucial for understanding border disputes between philosophers and psychologists. This difference is discussed at length in the exchange between Rey 1983, 1985, a philosopher, and Smith, Medin, and Rips 1984, psychologists all, and what follows borrows heavily from that exchange. Metaphysics deal with how the world is (which is investigated by science), while epistemology deals with how we know, believe, infer how the world is (Rey 1983). Thus, a metaphysical categorization considers what makes an entity an instance of a particular kind, whereas an epistomological categorization considers how an agent decides whether the entity is of a particular kind. We can illustrate with the concept gold (italics indicate concepts). A metaphysical categorization of an object with respect to gold would take atomic structure to be the ultimate criterion-because that is the best guess of the relevant science as to what the ultimate criterion is-whereas an epistemological categorization of an object with respect to gold might consider the object's color, value, and common usage.

It takes a bit more work to connect this distinction to the issues raised in the target text. Obviously, psychology deals with epistemological 
accounts, but why should it care about a metaphysical account of concepts? The reason is that, according to well-known arguments of Kripke (e.g. 1972) and Putnam (e.g. 1975), only a metaphysical account provides 'identity' conditions for concepts, i.e. conditions for deciding whether two concepts are the same or different. Thus, if you use an object's color and value to categorize it as gold, where I consider its customary use and where it is mined, we would not want to conclude that we have different concepts of gold. For, presumably, both of us recognize that the properties we are currently using to identify gold are not necessarily tied to gold. To get true identity conditions, we must move to the metaphysical level, and there the relevant science tells us that the best guess of an identity condition for gold is that it is a chemical element with atomic number 79; if this guess turns out to be right, you and I are both talking about gold only if we are both talking about the stuff with atomic number 79 .

The above is a rough version of the argument that Rey 1983 advances against a purely psychological (i.e. epistemological) approach to concepts. I think this argument provides a context in which to understand some of the philosophical complaints voiced in the target text, claims like ' . . concepts fall outside the domain of psychological processes', and 'The fundamental point is that grasp of a concept is a normative, rather than a descriptive, matter'. Such complaints may reduce to the claim that one must go outside of psychology to find true identity conditions of concepts, which means that psychology cannot supply a complete theory of concepts.

Even if the above argument is accepted at face value, it does not spell the end to a psychological (epistemological) study of concepts. As argued elsewhere (Smith et al. 1984), although psychology may not be able to supply a complete theory of concepts, it may be able to supply enough to account for many aspects of concepts and their use. For the notion of sameness of concepts, which is what psychologists presumably are unable to offer, may not be essential for elucidating many aspects of concept use. The ability of you and I to communicate about gold, for example, may not require that we have the same concepts, but rather only that we have similar mental contents, where the mental contents include properties that we use to identify gold as well as more diagnostic properties that we believe to be true of gold (such as, that there's something inside of gold that makes it look and act the way it does). More generally, similar mental contents may be sufficient to support a notion of communality of concepts, and communality may be all that is needed to account for important aspects of communication. So psychologists can accept the philosophers claims about the limits of a purely epistemological approach to concepts, yet still carve out a fruitful domain of study.

\section{Core versus Identification Procedure}

Within a psychological approach to concepts, one needs to distinguish two components of a concept: (1) the identification procedure, which consists of 
properties that are perceptually salient and easy to compute, though by no means perfectly diagnostic of concept membership; and (2) the core, which consists of properties that are more diagnostic of concept membership but that tend to be somewhat hidden and less accessible for rapid categorization (Smith and Medin 1981; Smith et al. 1984). I can illustrate with the concept bird. Identification properties might include flies, sings, perches in trees, and eats insects, whereas core properties might include having bird genes, or if you are not that sophisticated, having parents who were birds. Clearly, a property like flies is more salient than one like having bird genes, whereas the latter is the more diagnostic than the former.

This distinction has straightforward implications for some of the issues raised in the target text. The characterization of the so-called 'recognitional' approach to concepts ('Having the concept of a dog ... is principally a matter of being able to sort prototypical dogs from clear examples of nondogs') deals only with identification procedures and ignores cores. As a consequence, the recognitional approach is left wide-open to the linguists criticism that ' . . . an account in terms of prototypes must be able to allow for the fact that someone who has a concept of a grandfather knows that someone who is the father of a parent is a grandfather, whether he fits the prototype or not'. This criticism dissolves in the face of the identification procedure-core distinction: an account in terms of prototypes (i.e. identification procedures) need not worry about matters like the 'father of a parent is a grandfather' because that is the business of cores. Thus, the concept grandfather presumably contains identification properties like older, grey haired and kindly, as well as the core property of father of a parent; the former properties permit one to sort prototypical grandfathers from clear examples of non-grandfathers, whereas the latter property allows one to declare a father-of-a-parent a grandfather.

\section{Different Kinds of Concepts}

Sometimes the Forum discussion seems to presuppose that, psychologically speaking, all concepts are of the same sort. In contrast, a number of psychologists and linguists are currently arguing that there are qualitatively different kinds of concepts (e.g. Lakoff 1987; Smith 1989).

The most obvious difference in kind is that between 'classical' and 'prototype' concepts. Classical concepts are those whose core consists of a definition, i.e. a set of singly necessary and jointly sufficient properties. A standard example is bachelor, whose defining properties include adult, male, and unmarried. In contrast, the cores of prototype concepts do not consist of definitions, but rather of vague scientific-like beliefs (e.g. the core of bird includes bird-genes, whatever that is), and possibly a pointer to experts who could amplify and elaborate these beliefs (as suggested by Putnam 1975). Classical concepts are frequently parts of formal systems 
that are person-made (like the kinship system), whereas prototype concepts are frequently part of people's general knowledge about natural kinds and artifacts.

The Forum discussion happens upon this potential distinction but then dismisses it with the offhand remark that'... there are almost no concepts-apart from bachelor-for which definitions are available'. This is simply not the case. Classical concepts abound in formal systems that many people have knowledge about: consider square, circle, etc. from the system of geometry; odd number, even number, etc. from various systems of mathematics; robber, felon, etc. from the legal system; uncle, nephew, etc. from the kinship system; island, volcano, etc. from the geological system; and so on. In addition, many social concepts may have a classical structure. Consider concepts about national origin, such as German and Italian, where the core seems to come down to a few defining properties (e.g. either born in Germany or adopted citizenship in Germany). A similar story may hold for concepts of race (e.g. Black, White), gender (male, female), and profession (e.g. lawyer, baker). These social concepts are among the most widely used in categorizing other people. So there are plenty of cases of classical concepts, certainly enough to take seriously the idea that they constitute an important type of concept.

There seem to be needs to distinguish other kinds of concepts as well. Within the set of prototype concepts, there is some evidence that naturalkind concepts differ in important ways from artifact concepts (e.g. Gelman 1987; Malt 1985). And among social concepts, there is evidence that trait concepts like aggressive are different in kind from person concepts like aggressive person (Anderson and Klatzky, 1987). Any claims about a theory of concepts therefore needs to keep in mind just what kind concepts the theory is intended to apply to.

Department of Psychology University of Michigan

Ann Arbor

Michigan 48104-2994

\section{References}

Anderson, S.M. and Klatzky, R.L. 1987: Traits and Social Stereotypes: Levels of Categorization in Person Perception. Journal of Personality and Social Psychology, 53, 235-246.

Gelman, S.A. 1988: The Development of Induction Within Natural Kind and Artifact Categories. Cognitive Psychology, 20, 65-95.

Kripke, S. 1972: Naming and necessity. In D. Davidson and G. Harman (eds.), Semantics of Natural Language. Dordrecht: D. Reidel.

Lakoff, G. 1987: Women, Fire, and Dangerous Things. Chicago: University of 
Chicago Press.

Malt, B.C. 1985: Hedges and the Mental Representation of Categories. CC-AI: The Journal of the Integrated Study of Artificial Intelligence, Cognitive Science, and Applied Epistemology. 2, 13-23.

Putnam, H, 1975: The Meaning of 'Meaning'. In K. Gunderson (ed.), Language, Mind, and Knowledge. Minneapolis: University of Minnesota Press.

Rey, G. 1983: Concepts and Stereotypes. Cognition, 15, 237-262.

Rey, G. 1985: Concepts and Conceptions: A reply to Smith, Medin, and Rips. Cognition, 19, 297-303.

Smith, E.E. 1989: Concepts and Induction. In M.I. Posner (ed.), Foundations of Cognitive Science. Cambridge, MA: MIT Press.

Smith, E.E. and Medin, D.L. 1981: Categories and Concepts. Cambridge, MA: Harvard University Press.

Smith, E.E., Medin, D.L. and Rips, L.J. 1984: A Psychological Approach to Concepts: Comments on Rey's 'Concepts and Stereotypes'. Cognition, 17, 265-274. 